\title{
The implantable miniature telescope
}

\section{Envisioning a treatment for end-stage age-related macular degeneration}

\author{
Phillip Williams (Meds 2017), Steven Wong (Meds 2018) \\ Faculty Reviewer: Dr Alex Mao, MD, OD, MPH (Department of Ophthalmology)
}

\section{ABSTRACT}

Bilateral, end-stage, age-related macular degeneration (AMD) is a devastating condition of the eye. As the leading cause of blindness worldwide in the elderly, it leads to poor quality of life. While antivascular endothelium growth factor agents are used as front-line treatment for wet AMD, no current treatment exists for bilateral, end-stage AMD in Canada. The implantable miniature telescope (IMT), approved by the Food and Drug Administration in 2010, is a treatment option available for those over the age of $\mathbf{6 5}$ in the United States with stable severe to profound vision impairment (best-corrected visual acuity $20 / 160$ to $20 / 800$ ) caused by bilateral central scotomas associated with bilateral, end-stage AMD. Combining the fields of engineering and ophthalmology, intraocular implantation of the IMT provides improvements in patients' functional vision and quality of life. As a relatively new treatment targeting the elderly, there are strict inclusion criteria as well as risks associated with the procedure. However, research and continuing development in this field is ongoing to minimize these risks.

\section{AGE-RELATED MACULAR DEGENERATION}

Aging gracefully is a common goal amongst the elderly. Seniors over the age of 65 capable of performing activities of daily living (ADLs) such as walking and eating, as well as complex instrumental ADLs (IADLs), including driving and completing housework, report having a better quality of life, less morbidity, and less mortality. ${ }^{1,2}$ Vision plays arguably one of the most important roles in carrying out ADLs and IADLs, but unfortunately vision impairments are highly prevalent in the elderly population. ${ }^{2}$ Bilateral, end-stage, age-related macular degeneration (AMD) is one of the most debilitating ocular conditions that can dramatically impair the quality of life in seniors. ${ }^{3}$ It is a degenerative disease of the macula resulting in a loss of central vision, and central scotomas. ${ }^{4}$ As the leading cause of central vision loss worldwide for the elderly, ahead of cataracts, glaucoma, and diabetic retinopathy, AMD affects 0.9, 4.6, and 13.1 percent of people aged 65 to 74,75 to 84 , and $>84$, respectively. ${ }^{5}$ There are two types of AMD: dry AMD, which is characterized by the accumulation of yellow deposits in the macula called drusen, and wet/neovascular AMD, which can arise from dry AMD, and is characterized by damage to the macula due to neovascularization. ${ }^{3,4}$ While genetics may influence the development of wet AMD, current hypotheses highlight the involvement of the pro-angiogenic molecule vascular endothelial growth factor (VEGF) in this condition. ${ }^{3,6}$ Both dry and wet AMD can progress to end-stage AMD, which presents with bilateral, irreversible macular scars and/or large areas of degeneration of retinal pigment epithelium, which leads to profound vision loss, increased morbidity and a decreased quality of life. ${ }^{?}$

\section{CURRENT TREATMENTS}

Several treatment modalities are available for the management of AMD. Ranibizumab and bevacizumab are 2 Food and Drug Administration (FDA)-approved, anti-VEGF based agents used for the treatment of wet AMD. ${ }^{8}$ Ranibizumab is administered monthly by intravitreal injection, and demonstrates an acceptable safety profile. ${ }^{9}$ Ranibizumab has been shown to halt the progression of wet AMD and improve visual acuity by 3 lines on the Early Treatment Diabetic Retinopathy Study (ETDRS) scale in $30-40 \%$ of patients. ${ }^{10}$ However, anti-VEGF agents are ineffective for bilateral, end-stage AMD. ${ }^{10-12}$ An optical option to manage visual impairment is the usage of handheld Galilean or Keplerian telescopes. These handheld extraocular devices are cumbersome and have a restricted field of view, as vision depends more on head and hand movements than on natural eye movements. ${ }^{11}$ Currently, there is no approved treatment for bilateral, end-stage AMD in Canada.

\section{IMPLANTABLE MINIATURE TELESCOPES}

The implantable miniature telescope (IMT), approved by the United States FDA in 2010 for individuals over the age of 65 , is a treatment for bilateral, end-stage AMD that circumvents the problems associated with extraocular visual aids. ${ }^{11}$ This intraocular prosthetic is about the size of a pea (3.6 mm diameter, $4.4 \mathrm{~mm}$ length). It contains wide-angle micro-optical lenses in a Galilean telescope design, which when combined with the cornea of the eye, acts as a compound telescope. This effect causes retinal image enlargement of approximately 2.2 or 2.7 times, enabling an individual to be able to distinguish more detail from the visual field. ${ }^{13}$ The prosthetic is surgically placed monocularly into the capsular bag after removal of the eye's lens, protruding 0.1 to $0.5 \mathrm{~mm}$ from the pupillary plane. ${ }^{14}$ The IMT provides central vision to the eye by providing magnification and redirecting light onto the healthy perimacular areas of the retina. Coordinated use of the implanted eye for central vision and the fellow, non-operated eye for peripheral vision improves eyesight for patients with bilateral end-stage AMD, allowing them the possibility of participating in both stationary and dynamic activities. After the operation, patients are administered prednisolone acetate eyedrops for up to 3 months, along with a standardized 
course of antibiotics to facilitate recovery. ${ }^{14}$ Additionally, patients must agree to participate in postoperative visual training with a low vision specialist and occupational therapist to maximize their ability to perform daily activities. The rehabilitation, typically lasting 6 months to a year, involves training patients to perform tasks at different distances and improving hand-eye coordination in both stationary and dynamic postures. While this process is challenging, motivated patients with good psychosocial health and realistic goals are able to gain quality of life.

The IMT is well studied, and is the only FDA-approved telescopic prosthetic for the treatment of end-stage AMD. ${ }^{11}$ One year postimplantation, the IMT provides a 3-line or more improvement in both distance and near best-corrected visual acuity in $53 \%$ of patients, whereas the untreated fellow/contralateral eye showed improvements in $10 \%$ of patients. ${ }^{13}$ Using patient data over a period of 12 years, Brown et al showed that IMT implantation in patients with bilateral, end-stage AMD resulted in a higher quality of life gain compared to controls (fellow/contralateral eye which underwent cataract surgery), along with a cost-utility of \$19000 USD/ QALY $(<\$ 50000 \mathrm{USD} /$ QALY is considered to be very cost effective). ${ }^{15}$

\section{LIMITATIONS}

While the IMT can restore vision to some extent in patients with bilateral end-stage AMD, it cannot prevent the macula from further deterioration and is not considered a curative treatment. ${ }^{16}$ Thus, the IMT is not indicated for patients with active choroidal neovascular membranes. ${ }^{16}$ Another limitation of the IMT is that the surgical procedure to implant the IMT demands immense technical expertise, and therefore carries surgical risk. There has been a documented $20 \%$ decline in corneal health 3 months postoperatively, though damage to the cornea after this period is minimal. ${ }^{17}$ Nevertheless, excessive damage to the corneal endothelium may necessitate a corneal transplant. ${ }^{17}$ Due to the risks of performing this procedure, the IMT is contraindicated in individuals with prior intraocular or cornea surgery, low baseline corneal endothelium cell density, and/or other pathologic conditions affecting the retina. ${ }^{16}$ The surgical technique used to implant the IMT, along with the prosthetic itself, have undergone revisions to minimize the damage that occurs to the corneal endothelium. A recent 2013 study showed that the "next generation" telescopic prosthesis required smaller incisions to the eye than the current prosthetic, and that the resultant improvements in surgical technique led to less corneal endothelial damage. ${ }^{18}$ With regards to postsurgical events, the IMT is contraindicated in patients with cognitive impairment, as this may limit their ability to engage in the postoperative rehabilitation that patients receiving the prosthetic are required to attend. ${ }^{13,16}$ In addition to the rehabilitation process, patients with an IMT will still require glasses for further vision correction. ${ }^{16}$

\section{SUMMARY}

Bilateral, end-stage AMD is primarily a disease of the elderly. It results in both profound and irreversible visual impairment that can limit one's ability to engage in activities of daily living. The IMT is a prosthetic approved by the FDA in the United States for certain patients over the age of 65 with this condition. Implanted into the anterior compartment of the eye, the IMT provides an intricate means of improving visual acuity and quality of life for those with bilateral, end-stage AMD. Ongoing refinement to both the surgical technique and the IMT itself will address concerns over the risk of performing the implantation. While the IMT is not a cure, it is an improvement on the existing, handheld, extraocular devices used to manage end-stage AMD and offers some hope to people living with this debilitating condition.

\section{REFERENCES}

1. Millán-Calenti JC, Tubío J, Pita-Fernández S, González-Abraldes I, Lorenzo T, Fernández-Arruty T, et al. Prevalence of functional disability in activities of daily living (ADL), instrumental activities of daily living (IADL) and associated factors, as predictors of morbidity and mortality. Arch Gerontol Geriatr. 2010;50:306-10.

2. Kempen GIJM, Ballemans J, Ranchor A V., Van Rens GHMB, Zijlstra GAR. The impact of low vision on activities of daily living, symptoms of depression, feelings of anxiety and social support in community-living older adults seeking vision rehabilitation services. Qual Life Res. 2012;21:1405-11.

3. Lim LS, Mitchell P, Seddon JM, Holz FG, Wong TY. Age-related macular degeneration. Lancet. 2012; 379(9827):1728-38.

4. Jager RD, Mieler WF, Miller JW. Age-related macular degeneration. N Engl J Med. 2008;358(24):2606-17.

5. Smith W, Assink J, Klein R, Mitchell P, Klaver CCW, Klein BEK, et al. Risk factors for age-related macular degeneration: pooled findings from three continents. Ophthalmology. 2001;108(4):697-704.

6. Nowak JZ. Age-related macular degeneration (AMD): pathogenesis and therapy. Pharmacol Rep. 2006; 58:353-63.

7. Brown MM, Brown GC, Sharma S, Stein JD, Roth Z, Campanella J, et al. The burden of age-related macular degeneration: a value-based analysis. Curr Opin Ophthalmol. 2006;17(3):257-66.

8. Foss AJ, Childs M, Reeves BC, Empeslidis T, Tesha P, Dhar-Munshi $\mathrm{S}$, et al. Comparing different dosing regimens of bevacizumab in the treatment of neovascular macular degeneration: study protocol for a randomised controlled trial. Trials. 2015;16(1).

9. Rosenfeld PJ, Brown DM, Heier JS, Boyer DS, Kaiser PK, Chung CY, et al. Ranibizumab for neovascular age-related macular degeneration. N Engl J Med. 2006; 355(14):1410-31.

10. Rasmussen A, Sander B. Long-term longitudinal study of patients treated with ranibizumab for neovascular age-related macular degeneration. Curr Opin Ophthalmol. 2014;25(3):158-63.

11. Singer MA, Amir N, Herro A, Porbandarwalla SS, Pollard J. Improving quality of life in patients with end-stage age-related macular degeneration: focus on miniature ocular implants. Clin Ophthalmol. 2012;6:33-9. 
12. Cohen SY, Mimoun G, Oubraham H, Zourdani A, Malbrel C, Queré S, et al. Changes in visual acuity in patients with wet age-related macular degeneration treated with intravitreal ranibizumab in daily clinical practice: the LUMIERE study. Retina. 2013;33(3):474-81

13. Hudson HL, Lane SS, Heier JS, Stulting RD, Singerman L, Lichter PR, et al. Implantable miniature telescope for the treatment of visual acuity loss resulting from end-stage age-related macular degeneratio: 1-year results. Ophthalmology. 2006;113(11):1987-2001.

14. Colby KA, Chang DF, Stulting RD, Lane SS. Surgical placement of an optical prosthetic device for end-stage macular degeneration: the implantable miniature telescope. Arch Opthalmol. 2007; 125(8): 1118-21.

15. Brown GC, Brown MM, Lieske HB, Lieske PA, Brown KS, Lane SS. Comparative effectiveness and cost-effectiveness of the implantable miniature telescope. Ophthalmology. 2011;118:1834-43.

16. United States Food and Drug Administration. Summary of safety and effectiveness data. Food and Drug Administration (US); 2010 June 49 p. Report: PMA No. P050034

17. Bansal AS, Baker P, Haller JA. An implantable visual prosthetic for end-stage macular degeneration. Expert Rev Ophthalmol. 2011; 6(2): 141-5

18. Rosen E, Sachs D, Eliahu S Ben, Assia EI, Kleinmann G. Preclinical safety and stability study of a next generation telescope prosthesis for end-stage macular degeneration. Clin Exp Ophthalmol. 2013;41:491-9.

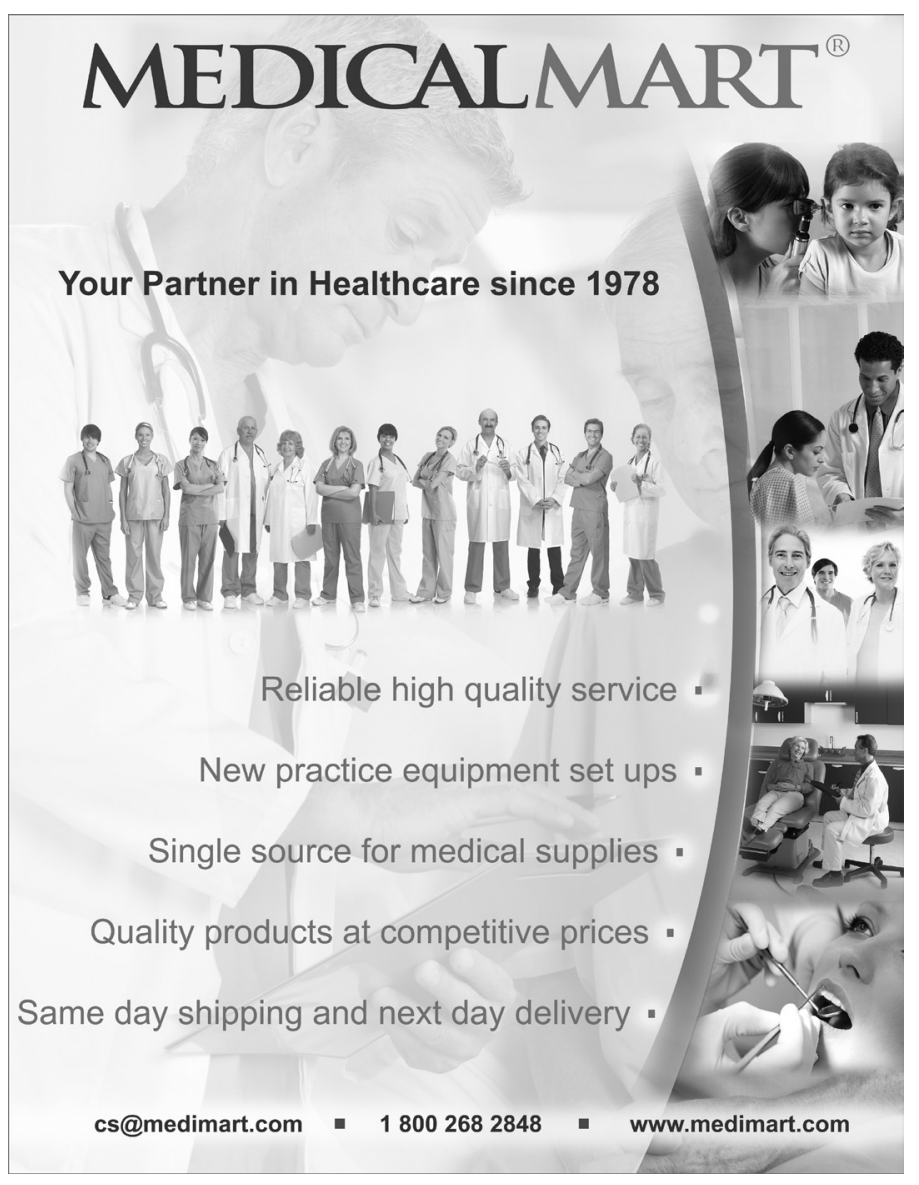

